\title{
Representation of macroscopic magnetization based on bifurcation property of domain structure
}

\section{$\operatorname{AUTHOR}(\mathrm{S})$ :}

Nakamura, T.; Ito, S.; Mifune, T.; Matsuo, T.; Kaido, C.

\section{CITATION:}

Nakamura, T....[et al]. Representation of macroscopic magnetization based on bifurcation property of domain structure. Journal of Applied Physics 2015, 117(17): 17 E516.

\section{ISSUE DATE:}

2015-05-07

URL:

http://hdl.handle.net/2433/198438

\section{RIGHT:}

(C) 2015 American Institute of Physics. This article may be downloaded for personal use only. Any other use requires prior permission of the author and the American Institute of Physics. 


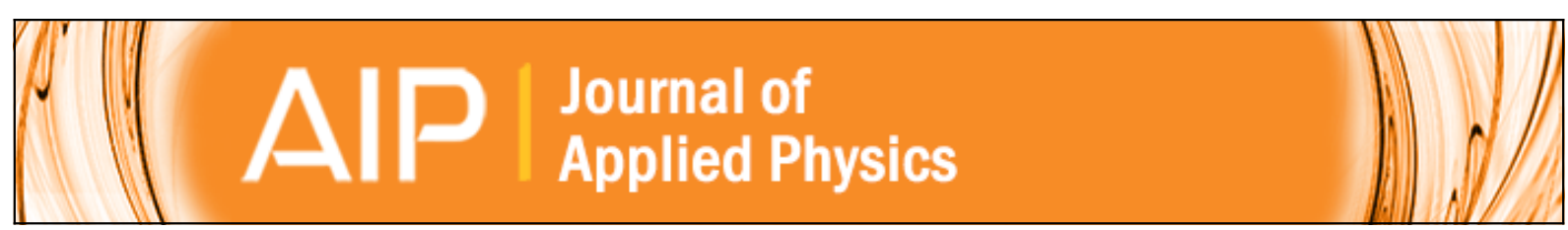

\section{Representation of macroscopic magnetization based on bifurcation property of domain structure}

T. Nakamura, S. Ito, T. Mifune, T. Matsuo, and C. Kaido

Citation: Journal of Applied Physics 117, 17 E516 (2015); doi: 10.1063/1.4918637

View online: http://dx.doi.org/10.1063/1.4918637

View Table of Contents: http://scitation.aip.org/content/aip/journal/jap/117/17?ver=pdfcov

Published by the AIP Publishing

\section{Articles you may be interested in}

Size effect on the structural, magnetic, and magnetotransport properties of electron doped manganite La0.15Ca0.85MnO3

J. Appl. Phys. 111, 07D729 (2012); 10.1063/1.3680246

Magnetic study of the Co-MCM-41 catalyst: Before and after reaction

J. Appl. Phys. 110, 103904 (2011); 10.1063/1.3660775

Magnetic properties of Fe 75 Si 15 Al 10 crystalline powders and domain structural observations using Lorentz transmission electron microscopy

J. Appl. Phys. 103, 07E719 (2008); 10.1063/1.2831365

Magnetic and optical properties of ionic ferrofluids based on nickel ferrite nanoparticles

J. Appl. Phys. 88, 6628 (2000); 10.1063/1.1288016

Reorientational magnetic transition in high-density arrays of single-domain dots

Appl. Phys. Lett. 76, 3609 (2000); 10.1063/1.126722

Frustrated by

old technology?

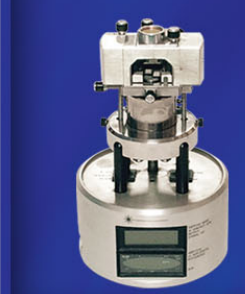

Is your AFM dead

and can't be repaired?

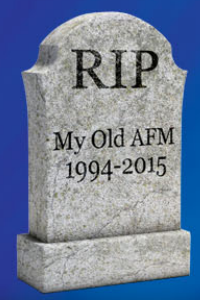

Sick of bad customer support?

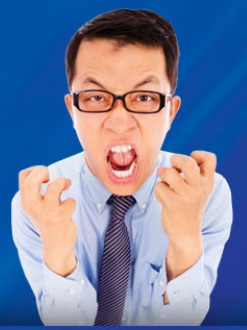

It is time to upgrade your AFM Minimum \$20,000 trade-in discount for purchases before August 31st

Asylum Research is today's technology leader in AFM

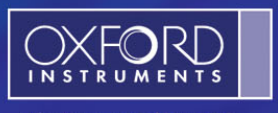




\title{
Representation of macroscopic magnetization based on bifurcation property of domain structure
}

\author{
T. Nakamura, ${ }^{1}$ S. Ito, ${ }^{1}$ T. Mifune, ${ }^{1}$ T. Matsuo, ${ }^{1, \text { a) }}$ and C. Kaido ${ }^{2, b)}$ \\ ${ }^{1}$ Graduate School of Engineering, Kyoto University, Kyoto daigaku-katsura, Nishikyo-ku, Kyoto 615-8510, \\ Japan \\ ${ }^{2}$ Department of Electrical and Electronic Engineering, Kitakyushu National College of Technology, \\ 5-20-1 Shii, Kokuraminamiku, Kitakyushu, Fukuoka 802-0985, Japan
}

(Presented 5 November 2014; received 22 September 2014; accepted 21 December 2014; published online 17 April 2015)

This study presents an efficient representation of macroscopic magnetization process based on the bifurcation property of mesoscopic two-domain magnetic particles. The macroscopic magnetization model, called the assembled domain structure model (ADSM), is constructed by assembling mesoscopic particles. Bifurcation is discussed of the two-domain state from the single-domain state in the unit mesoscopic particle in the ADSM. Based on this state bifurcation, a procedure to switch the macroscopic magnetization-state transition in the ADSM is developed, which dramatically reduces computation times in macroscopic magnetization simulations. @ 2015 AIP Publishing LLC.

[http://dx.doi.org/10.1063/1.4918637]

\section{INTRODUCTION}

Macroscopic magnetic properties of iron-core materials are governed by multiscale magnetization processes such as microscopic domain-wall motion and mesoscopic domainstructure transition. To construct a physical macroscopic magnetization model, several energy-based multiscale approaches have been proposed. Taking account of the magnetoelastic effect and assuming the magnetic-domain equilibrium based on the probability function, ${ }^{1}$ Ref. 2 has succeeded in reconstructing anhysteretic magnetic and magneto-mechanical properties of grain-oriented silicon steel. In Ref. 3, using a local energy minimization approach, an assembly of mesoscopic two-domain magnetic particles represents the macroscopic hysteretic behavior of a magnetic sheet.

However, these multiscale approaches often require long computational times. The former model has large computational complexity in handling stress-dependent crystalgrain behavior. The latter, here called the assembled domain-structure model (ADSM), exhibits inefficiencies in calculating the minimum local energy causing slow convergence to/escape from equilibrium points during the transition of magnetization states such as in magnetization reversal.

This study presents an efficient procedure to accelerate calculations of the magnetization-state transition. The procedure is based on the bifurcation property of two-domain magnetic particles. First, the bifurcation of the two-domain state from the single-domain state in the unit particle in the ADSM is discussed. Based on the bifurcation property above, the switching procedure for the magnetization-state transition in the ADSM is developed.

\footnotetext{
${ }^{\text {a)} E l e c t r o n i c ~ m a i l: ~ t m a t s u o @ k u e e . k y o t o-u . a c . j p . ~}$

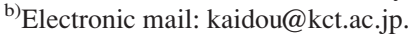

\section{DOMAIN STRUCTURE MODEL}

\section{A. Simplified domain structure model (SDSM)}

This paper uses the $\mathrm{SDSM}^{4,5}$ having two domains as in Fig. 1(a), labeled $i=1,2$, each having uniform magnetization. The normalized magnetization vectors in the domains are labeled $\boldsymbol{m}(i)=\left(\sin \theta_{i} \cos \phi_{i}, \sin \theta_{i} \sin \phi_{i}, \cos \theta_{i}\right)$. The magnetization state of the SDSM is represented by the state vector $\boldsymbol{X}=\left(\theta_{1}, \phi_{1}, \theta_{2}, \phi_{2}, \lambda\right)$, where $\lambda$ is the volume ratio of domain 1 . The total magnetic energy to be minimized is normalized by the crystalline anisotropy energy. The normalized total magnetic energy, $e$, is given as

$$
e=e_{\mathrm{ap}}+e_{\mathrm{an}}+e_{\mathrm{w}}+e_{\mathrm{st}},
$$

where $e_{\text {ap }}$ is the Zeeman energy, $e_{\text {an }}$ is the crystalline anisotropic energy, $e_{\mathrm{w}}$ is the domain-wall energy, and $e_{\mathrm{st}}$ is the magnetostatic energy. The normalized crystalline anisotropic energy is given as

$$
e_{\mathrm{an}}=\lambda f_{\mathrm{an}}\left(\theta_{1}, \phi_{1}\right)+(1-\lambda) f_{\mathrm{an}}\left(\theta_{2}, \phi_{2}\right),
$$

where $f_{\text {an }}$ represents the angular dependence. The Zeeman energy associated with the normalized applied field, $\boldsymbol{h}=h\left(\cos \phi_{\text {ap }}, \sin \phi_{\text {ap }}\right)$, is given as

$$
e_{\mathrm{ap}}=-2 \boldsymbol{h} \cdot\left[\lambda \boldsymbol{m}_{1}+(1-\lambda) \boldsymbol{m}_{2}\right],
$$

where $h=H_{\text {ap }} /\left(\kappa M_{\mathrm{S}}\right), H_{\text {ap }}$ is the magnitude of the applied magnetic field, $M_{\mathrm{S}}$ is the magnitude of the spontaneous magnetization, $\kappa=2 K /\left(\mu_{0} M_{\mathrm{S}}^{2}\right)$, and $K$ is the anisotropy constant. A simple Bloch wall model gives the domain-wall energy as

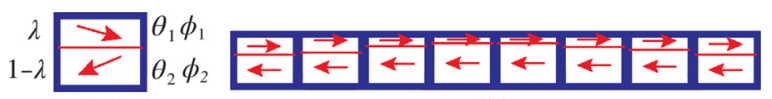

(a)

(b)

FIG. 1. 1D alignment of cells. 


$$
e_{\mathrm{w}}=w\left(1-\boldsymbol{m}_{1} \cdot \boldsymbol{m}_{2}\right) / 2,
$$

where $w=4 l_{k} / D, l_{k}=\sqrt{A / K}, D$ is the width of the two domains, and $A$ is the exchange stiffness constant. The SDSM assumes that the demagnetizing field is uniformly given by the multiplication of the demagnetizing factors and the average magnetization. The magnetostatic energy is given as

$$
e_{\mathrm{st}}=s_{x} m_{x}^{2}+s_{y} m_{y}^{2}+s_{z} m_{z}^{2},
$$

where $\left(m_{x}, m_{y}, m_{z}\right)=\boldsymbol{m}=\lambda \boldsymbol{m}_{1}+(1-\lambda) \boldsymbol{m}_{2}, s_{x}=k_{x} / \kappa, s_{y}$ $=k_{y} / \kappa$, and $s_{z}=k_{z} / \kappa ; k_{x}, k_{y}$, and $k_{z}$ are the demagnetizing factors.

The necessary condition for the total energy to be minimized locally is given by $\partial e / \partial \boldsymbol{X}=0$. In Refs. 4 and 5 , the magnetization state is determined by the direct solution of this equation, either numerically or analytically. In Ref. 3, a local energy minimum is obtained by the temporal integration of the following artificial state equation integrated up to a stable equilibrium point:

$$
\frac{\mathrm{d} \boldsymbol{X}}{\mathrm{d} t}=\boldsymbol{Y}, \quad \frac{\mathrm{d} \boldsymbol{Y}}{\mathrm{d} t}=-\frac{\partial e}{\partial \boldsymbol{X}}-\beta \boldsymbol{Y},
$$

where $\beta \boldsymbol{Y}$ is the dissipation term, and $\beta$ is the dissipation coefficient.

\section{B. Bifurcation property in SDSM}

For simplicity, an in-plate rotation of the magnetization vectors is assumed where $\theta_{1}$ and $\theta_{2}$ are fixed to $\pi / 2$ and $\phi_{1}$ and $\phi_{2}$ are the rotating variables.

The solution satisfying $\phi_{1}=\phi_{2}$ means the singledomain state, where $\partial e / \partial \boldsymbol{X}=0$ requires

$$
\frac{\partial f_{\mathrm{an}}(\phi)}{\partial \phi}+2 h \sin \left(\phi-\phi_{\mathrm{ap}}\right)+\left(s_{y}-s_{x}\right) \sin 2 \phi=0 .
$$

This subsection assumes that $\phi_{\text {ap }}$ is fixed and discusses the bifurcation resulting from the variation in $h$. When $h \rightarrow \infty$, a stable solution $\phi_{1}=\phi_{2}=\phi_{\text {ap }}$ exists, which loses stability when $h$ becomes small.

The stability of the solution is evaluated using the eigenvalues of the Hessian matrix of $e$ given as

$$
\frac{\partial^{2} e}{\partial \boldsymbol{X}^{2}}=\left(\begin{array}{ccc}
\lambda F-C & C & 0 \\
C & (1-\lambda) F-C & 0 \\
0 & 0 & 0
\end{array}\right)
$$

where

$$
\begin{gathered}
F=\frac{\partial^{2} f_{\mathrm{an}}(\phi)}{\partial \phi^{2}}+2 h \cos \left(\phi-\phi_{\mathrm{ap}}\right)+2\left(s_{y}-s_{x}\right) \cos 2 \phi \\
C=\lambda(1-\lambda)\left[s_{x}+s_{y}+\left(s_{y}-s_{x}\right) \cos 2 \phi\right]-w / 2
\end{gathered}
$$

One eigenvalue of $\partial^{2} e / \partial \boldsymbol{X}^{2}$ is obviously zero, which implies that $\lambda$ does not affect the single domain solution. The solution of (7) is stable if the other two eigenvalues are positive. A bifurcation occurs when a second eigenvalue becomes 0 , which implies

$$
F=s_{x}+s_{y}+\left(s_{y}-s_{x}\right) \cos 2 \phi-\frac{w}{2 \lambda(1-\lambda)} \text { or } 0 .
$$

Equation (10) implies

$$
h=h_{\mathrm{S}}(\lambda) \text { or } h_{\mathrm{s}},
$$

where

$$
\begin{gathered}
h_{\mathrm{S}}(\lambda)=\frac{-\frac{\partial^{2} f_{\mathrm{an}}(\phi)}{\partial \phi^{2}}-\left(s_{y}-s_{x}\right) \cos 2 \phi+s_{x}+s_{y}-\frac{w}{2 \lambda(1-\lambda)}}{2 \cos \left(\phi-\phi_{\mathrm{ap}}\right)}, \\
h_{\mathrm{s}}=-\frac{\frac{\partial^{2} f_{\mathrm{an}}(\phi)}{\partial \phi^{2}}+2\left(s_{y}-s_{x}\right) \cos 2 \phi}{2 \cos \left(\phi-\phi_{\mathrm{ap}}\right)} .
\end{gathered}
$$

Consequently, the single-domain solution is stable when

$$
h \geq \max \left(h_{\mathrm{S}}(\lambda), h_{\mathrm{s}}\right) .
$$

Whereas $h_{\mathrm{s}}$ does not depend on $\lambda$, the maximal $h_{\mathrm{S}}(\lambda)$ occurs when $\lambda=1 / 2$ giving value $h_{\mathrm{S}}(1 / 2)$ for which the solution can become unstable. For $h=h_{\mathrm{s}}$, the eigenvector for the zero eigenvalue is $(\Delta \phi, \Delta \phi, 0)$, corresponding to the bifurcation of an unstable solution of the coherent rotation with $\phi_{1}=\phi_{2}$. For $h=h_{\mathrm{S}}(1 / 2)$, the eigenvector for the zero eigenvalue is $(\Delta \phi,-\Delta \phi, 0)$, corresponding to the bifurcation of a two-domain solution with $\phi_{1} \neq \phi_{2}$.

Therefore, the transition from the single-domain solution can be checked using the eigenvalues of $\partial^{2} e / \partial \boldsymbol{X}^{2}$ with $\lambda=1 / 2$.

The transition from a two-domain to a single-domain solution can be accelerated also by checking the eigenvalues of $\partial^{2} e / \partial \boldsymbol{X}^{2}$ as addressed in Sec. III B.

\section{ASSEMBLY OF SDSMs}

\section{A. Assembled domain structure model}

The macroscopic magnetization model is constructed from an assembly of SDSMs, which is called the ADSM hereafter. Each SDSM composing the ADSM is called a cell. The Zeeman energy, the anisotropic energy, and the domainwall energy of cells are independently summed up to obtain the energy components of the ADSM. The exchange interaction between the cells is not taken into account.

The demagnetization field is given by the summation of the dipole field generated by the average magnetization of every cell. The demagnetization field computation results in the convolution of demagnetization coefficient matrices and magnetization vectors in a similar manner to the micromagnetic simulation as ${ }^{5}$

$$
\boldsymbol{h}_{\mathrm{st}}(i)=\sum_{i^{\prime}} \boldsymbol{s}\left(i-i^{\prime}\right) \boldsymbol{m}\left(i^{\prime}\right)
$$

where $\boldsymbol{h}_{\mathrm{st}}$ is the normalized demagnetization field, $i$ and $i^{\prime}$ are cell indexes, $s\left(i-i^{\prime}\right)$ is the normalized demagnetizing coefficient matrix between the cells $i$ and $i^{\prime}$, and $\boldsymbol{m}(i)$ is the normalized magnetization of the cell $i$. 
The state variable vector $\boldsymbol{X}$ consists of $\boldsymbol{X}(i)(i=1, \ldots)$ in each cell. A local energy minimum point is obtained by solving Eq. (6).

\section{B. Assembled domain structure switching model (ADSSM)}

The macroscopic magnetization reversal in the ADSM is represented by the sequence of magnetization reversals of each unit cell. However, the transient process required to reach an equilibrium point of Eq. (6) may take a long time. From the bifurcation behavior outline above, this subsection develops a method to accelerate the magnetization state transition by switching the magnetization states of the unit cells discontinuously one by one, hereafter referred to as the ADSSM.

The ADSSM has the same energy components as the ADSM. The demagnetizing field $\boldsymbol{h}_{\mathrm{st}}$ in a cell is divided into two components $\boldsymbol{h}_{\text {stin }}$ and $\boldsymbol{h}_{\text {stex }}$ that are generated by the cell itself and by the other cells, respectively. They are given as

$$
\begin{gathered}
\boldsymbol{h}_{\text {stin }}(i)=\boldsymbol{s}(0) \boldsymbol{m}(i), \\
\boldsymbol{h}_{\text {stex }}(i)=\sum_{i^{\prime} \neq i} \boldsymbol{s}\left(i-i^{\prime}\right) \boldsymbol{m}\left(i^{\prime}\right) .
\end{gathered}
$$

By including $\boldsymbol{h}_{\text {stex }}$ into the applied field, bifurcation can be applied to each cell where $\operatorname{diag}\left(s_{x}, s_{y}, s_{z}\right)=s(0)$. The applied magnetic field $\boldsymbol{h}=h\left(\cos \phi_{\text {ap }}, \sin \phi_{\text {ap }}\right)$ is replaced by the effective applied magnetic field $\boldsymbol{h}_{\text {eff }}$ given as

$$
\boldsymbol{h}_{\text {eff }}=\boldsymbol{h}+\boldsymbol{h}_{\text {stex }} .
$$

The unit cell has either the single domain or the twodomain state. If the $i$-th cell has the single-domain state, the state vector $\boldsymbol{X}(i)$ satisfies Eq. (7). If $\phi_{\text {ap }}=0, \boldsymbol{X}(i)$ is given as

$$
\boldsymbol{X}(i)=\left(\frac{\pi}{2}, 0, \frac{\pi}{2}, 0, \frac{1}{2}\right) \text { or }\left(\frac{\pi}{2}, \pi, \frac{\pi}{2}, \pi, \frac{1}{2}\right) .
$$

When $\phi_{\mathrm{ap}}=0$, the two-domain state is given as

$$
\boldsymbol{X}(i)=\left(\frac{\pi}{2}, 0, \frac{\pi}{2}, \pi, \frac{1}{2}\left(1+\frac{h}{s_{x}}\right)\right)
$$

where the crystalline easy axis is assumed along the $x$ direction.

Alternating magnetization is obtained by changing the applied field $h$ step by step to find a corresponding equilibrium point, described as follows. For each cell, all eigenvalues of the Hessian matrix are calculated. If one of the eigenvalues in a cell becomes negative, it is possibly that the cell changes its magnetization state. Such cells are hereafter referred to as possibly unstable cell, whereas cells having no negative eigenvalue are called the stable cell. Among the possibly unstable cells, the cell having the largest magnitude among the negative eigenvalues is chosen as the most unstable cell to undergo the state transition. The equilibrium point of the most unstable cell is searched by integrating the artificial state equation (6). The initial values of the state variables are set to a predicted equilibrium point such as given by
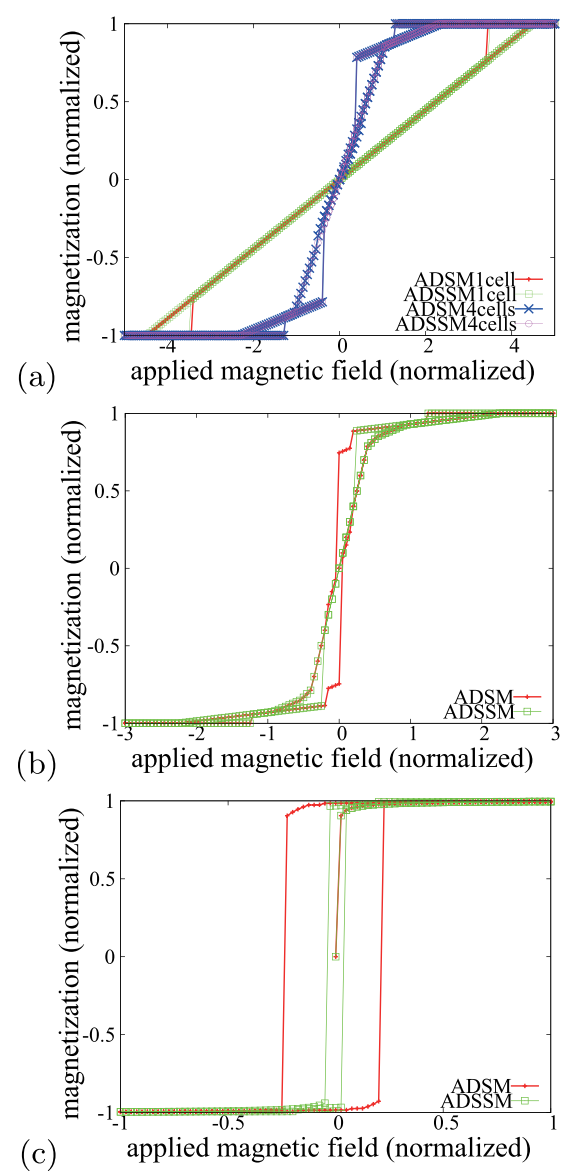

FIG. 2. Simulation results of the ADSM and ADSSM with the number of cells of (a) 1 and 4, (b) 8, and (c) 128 .

Eq. (18) for the transition from the single to two-domain state and Eq. (19) for the reverse transition. In accordance with the change of the effective applied field, the equilibrium point of every stable cell is also corrected independently from other cells. After the state transition of the most unstable cell, the demagnetizing field is recalculated and the procedure above is repeated until all cells attain their stable equilibrium points.

\section{MAGNETIZATION ANALYSIS UNDER ALTERNATING MAGNETIC FIELD}

The cells aligned one-dimensionally, as in Fig. 1(b), are magnetized along their longitudinal directions. The normalized cell size is $1 \times 1 \times 0.1$, where $\kappa=0.01, w=0.01$, and $\boldsymbol{s}(0)=\operatorname{diag}(4.48,4.48,91.0)$. For example, those normalized

(a)

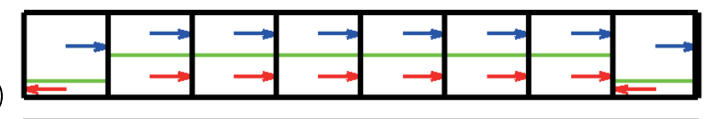

(b)

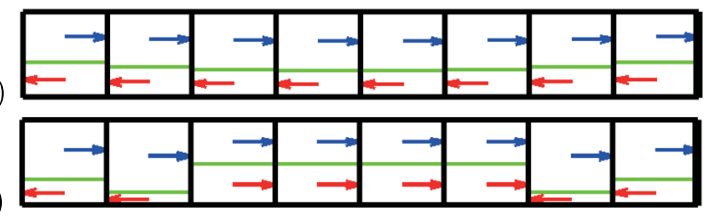

FIG. 3. Magnetization states of 8 cells obtained by (a) the ADSSM at $h=0.5$, (b) the ADSSM at $h=0.15$, and (c) the ADSM at $h=0.15$. 
TABLE I. Computation time (s) for ADSSM and ADSM.

\begin{tabular}{lccc}
\hline \hline Number of cells & 1 & 8 & 128 \\
\hline ADSSM & 0.234 & 4.79 & 48.11 \\
ADSM & 5.32 & 134.4 & 3511 \\
\hline \hline
\end{tabular}

parameters are obtained from artificial material parameters of $\mu_{0} M_{\mathrm{S}}=2.2 \mathrm{~T}, K=1.9 \times 10^{4} \mathrm{~J} / \mathrm{m}^{4}, A=1.2 \times 10^{-11} \mathrm{~J} / \mathrm{m}$, $D=10^{-5} \mathrm{~m}$, and the cell size of $D \times D \times 0.1 D$. Fig. 2 shows the magnetization curves obtained by the ADSM and ADSSM using 1, 4, 8, and 128 cells. The magnetization curves obtained by the ADSSM coincide with those obtained by the ADSM in the cases of 1 and 4 cells.

For 128 cells, however, the coercivity given by the ADSM is larger than that obtained by the ADSSM. This suggests that the ADSM sometimes fails to assess convergence correctly and in error stops the time-integration of Eq. (6) before escape from an unstable equilibrium point becomes evident because the transient time is very long. Figure 3 illustrates the spatial distribution of magnetization states of 8 cells obtained by the ADSSM and ADSM. The comparison of Fig. 3(b) with Fig. 3(c) shows that the ADSSM promotes the transition from the single-domain state to the twodomain state more strongly than the ADSM. As a result, the ADSSM reconstructs the very small coercive force compared with the anisotropy field that is often observed in soft magnetic materials.

Table I lists the computation time required by both models in obtaining the magnetization curve where the ADSSM reduces the computation time to $1 / 73$ of that consumed by the ADSM for 128 cells.

For comparison, Fig. 4 shows the magnetization curves yielded by the micromagnetic simulation solving the LandauLifshitz-Gilbert (LLG) equation, where the material parameters correspond to those for the ADSM/ADSSM. The material size of $4 D \times D \times 0.1 D$ is divided into $128 \times 32 \times 1,256$ $\times 64 \times 1$, and $512 \times 128 \times 1$ square prism cells. The time integration of LLG equation is executed until the normalized magnetization change $|\partial \boldsymbol{m} / \partial t|$ becomes less than $10^{-5}$ in all the cells. The magnetization curves roughly agree with those given by the ADSM/ADSSM.

Figure 5 shows the magnetization curves obtained by the ADSSM where the sample size is fixed as $128 D \times D \times 0.1 D$, which is divided into $1,16,32,64$, and 128 cells along the longitudinal direction. In the cases of 1 , 16,32 , and 64 cells, the shape anisotropy $\left(s_{x}<s_{y}\right)$ in the unit cell results in the large coercive force, which is similarly obtained by the ADSM.

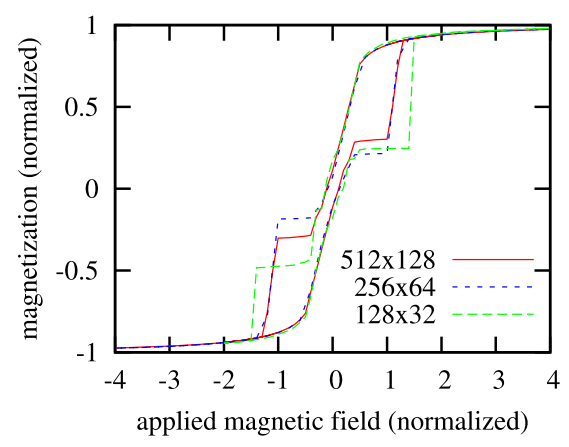

FIG. 4. Magnetization curves given by micromagnetic simulation with $128 \times 32 \times 1,256 \times 64 \times 1$, and $512 \times 128 \times 1$ cells.

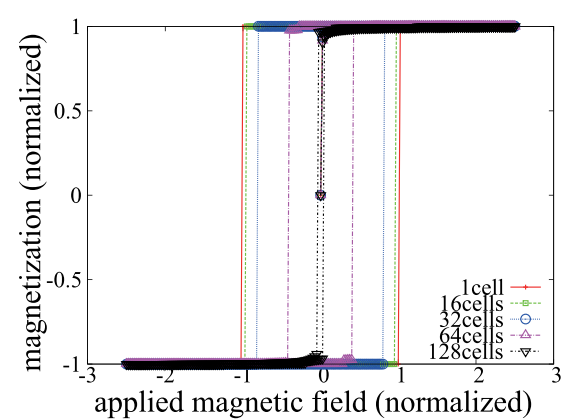

FIG. 5. Magnetization curves given by the ADSSM with the fixed sample size of $128 \times 1 \times 0.1$ divided into $1,16,32,64$, and 128 cells.

\section{CONCLUSION}

An efficient representation of macroscopic magnetization is achieved by using the bifurcation of mesoscopic particles. By accelerating the change in the states of magnetization, the proposed model reduces the computation time to $1 / 73$ of macroscopic models without acceleration.

\section{ACKNOWLEDGMENTS}

This work was supported in part by the Japan Society for the Promotion of Science, Grant-in-Aid for Scientific Research (C), Grant No. 26420232.

${ }^{1}$ L. Daniel, O. Hubert, N. Buiron, and R. Billardon, "Reversible magnetoelastic behavior: A multiscale approach," J. Mech. Phys. Solids 56, 1018-1042 (2008).

${ }^{2}$ O. Hubert and L. Daniel, "Multiscale modeling of the magneto-mechanical behavior of grain-oriented silicon steels," J. Magn. Magn. Mater. 320, 1412-1422 (2008).

${ }^{3}$ M. Sudo, T. Mifune, T. Matsuo, and C. Kaido, "A simplified domain structure model exhibiting the pinning field," IEEE Trans. Magn. 49, 1829-1832 (2013).

${ }^{4}$ T. Matsuo, "Magnetization process analysis using a simplified domain structure model,” J. Appl. Phys. 109, 07D332 (2011).

${ }^{5} \mathrm{M}$. Sudo and T. Matsuo, "Magnetization modeling of silicon steel using a simplified domain structure model,” J. Appl. Phys. 111, 07 D107 (2012). 